\title{
Nanotecnologías, tierras raras y ética: la defensa por un giro ético
}

\begin{abstract}
Fanny VerRax*
Resumen: Este trabajo explora los vínculos entre las nanotecnologías y los elementos de tierras raras desde un punto de vista ético. Mientras el campo de la nanoética emerge como un área robusta con muchos estudios de académicos trabajando sobre aspectos éticos y sociales asociados con las nanotecnologías, los elementos de tierras raras han sido en cambio raramente abordados por filósofos y eticistas. El presente artículo sostiene que este tipo de dicotomía en términos de intereses investigativos ilustra una tendencia desafortunada hoy en día en el campo de la ética aplicada que prefiere enfocarse en preguntas sobre "¿y si?" en lugar de aquellas sobre cuestiones del "aquí y el ahora". En tal sentido, se dibuja un paralelismo entre esta tendencia y la bien conocida dicotomía entre la filosofía moral y la moralidad y la contextualización. Se propone esto último para apreciar los aspectos éticos que la tecnociencia acarrea.

PalABRAS ClaVE: tierras raras, nanotecnología, tecnociencia, moral, ética.

ABSTRACT: This paper explores the links between nanotechnologies and rare earth elements from an ethical point of view. While the field of nanoethics has emerged as a well-investigated area with many scholars addressing ethical and social issues raised by nanotechnologies, rare earth elements have been rather understudied by philosophers and ethicists. The paper claims that this dichotomy in terms of research interest illustrates an unfortunate tendency in the field of applied ethics today that prefers to focus on "what if?" questions rather than on "hic and nunc" issues. I draw a parallel between this tendency and the well-known dichotomy in moral philosophy between morality and situatedness, and propound to use the latter in our grasping of ethical issues raised by technoscience.

KEY wORDS: rare earth, nanotechnology, tecnoscience, morality, ethics.
\end{abstract}

\section{NanoÉtica: el SURgimiento del “ ¿Y SI?”}

La nanoética se ha convertido en un área académica bien establecida y las nanotecnologías han "causado el nacimiento de una rama entera de la ética" (Nurock, 2010: 31). ${ }^{1}$ La nanoética disfruta incluso de su propia revista dictaminada, Nanoethics, publicada por Springer desde 2007. Esta misma revista, interdisciplinaria, ofrece muchas miradas éticas como parte necesaria de la reflexión en torno a las nanotecnologías. Los aspectos nanoéticos incluyen típicamente aquellos alrededor de la privacidad y el control, la percepción pública de las nanotecnologías en diferentes áreas geográficas y contextos culturales, o cuestiones éticas derivadas de distintas aplicaciones nanotecnológicas (en biología sintética, nanomedicina, nanotubos, entre otras). Algunos

\footnotetext{
* Estudiante postdoctoral de la Escuela Normal Superior de Lyon; su línea de investigación se enfoca en la "antropología del reciclado de materiales estratégicos". Su principal formación es en filosofía de la ciencia y estudios socioambientales. (Fanny.verrax@ens-lyon.fr).

1 Uno debe tener en cuenta, sin embargo, que la "singularidad del debate" no se resuelve de una vez por todas. Véase: Grunwald (2005).
} 
autores han explicado exitosamente por qué la ética ha sido un componente importante en el desarrollo de las nanotecnologías desde el surgimiento (Strand y Nydal, 2008) de la reacción social causada por los OGMs en Europa, la cual llevó a reconocer la necesidad de una "coproducción" de ciencia, tecnología y sociedad (Jasanoff, 2004). Esto es, sin embargo, cierto también para el caso de otras tecnologías emergentes, por lo que no se podría explicar la infatuación particular por la nanoética. Así las cosas, ahora será la especificidad de la nanoética explorada y sugeriré una hipótesis: específicamente, la razón por la cual los aspectos éticos han recibido mayor atención en el campo de las nanotecnologías podría ser el tratarse de una área que abre todo tipo de imaginarios, convirtiéndose así en un campo de juego lleno de posibilidades para los eticistas. Las perspectivas en nanoética nos llevarán a distinguir entre tres clases de ética, dependiendo del nivel de imaginación involucrado: normal, futurista y al que denomino ética ficción. Mientras sería delicado trazar una delgada línea entre la segunda y la tercera modalidad, ambas pueden considerarse como la respuesta ética a la clase de cuestionamientos iniciados con el "¿y si?".

Pero, por qué las nanotecnologías estimulan particularmente la imaginación? Un aspecto puede asociarse al "embrollo de ilustrar e imaginar" (Ruivenkamp y Rip, 2011) la nanoescala, al ser por definición invisible; visualizar e ilustrar siempre involucran algún tipo de habilidades imaginativas, representando usualmente lo que podría ser en lugar de lo que es.

[...] uno puede distinguir entre ilustrar, esto es, crear imágenes basadas en información y enfocadas a rensamblar, de la imaginación, donde ésta es movilizada para crear una visión del nanomundo. De hecho, ilustrar e imaginar siempre están propiamente entretejidas, en las denominadas visualizaciones científicas, donde las expectativas acerca de cómo la nanoescala podría verse dirigen las opciones involucradas en el quehacer de las imágenes. (Ruivenkam y Rip, 2012: 185)

Este enfoque sobre los futuros potenciales, en lugar de ser sobre el desarrollo real, podría explicar la tendencia de muchos nanoéticistas de explorar escenarios, haciendo de la nanoética la esfera del "¿y si?".

Esta tendencia parece compartida igualmente por ambos proponentes y oponentes de las nanotecnologías. Los primeros enfocándose en los beneficios esperados (¿milagros?) de las nanotecnologías (abastecimiento universal de agua potable, mejor y más barata generación de energía, alimentos interactivos inteligentes, etc.); los segundos más centrados en los riesgos - es interesante notar que esos riesgos pertenecen a las mismas "áreas" en las que suponen estar los beneficios supuestamente a alcanzar: la salud, el ambiente y los aspectos sociales-. Esos tres dominios de preocupación convergen algunas veces en escenarios catastróficos, tales como la hipótesis de la denominada "plaga gris" (grey goo) o de la replicación de nanosistemas fuera de control. En este escenario, los robots autorreplicantes, construidos a partir de nanotecnologías moleculares, consumen toda la materia del planeta mientras continúan reproduciéndose, llevando así al fin del mundo. ${ }^{2}$

Esto obliga a dar cuenta de la necesidad de distinguir en la nanoética entre nanotecnologías "normales" y "futurísticas", tal y como lo ha teorizado Donlad O'Mathuna (2009).

2 Véase, por ejemplo:<www.wired.com/medtech/health/news/2004/07/64235>. 
La "nanotecnología normal" refiere a la investigación, desarrollo y producción ordinaria de transistores, fármacos, materiales nanoestructurados, etc., que se ha gestado en décadas. La "nanotecnología futurística" alude al mundo especulativo de los "ensambladores moleculares", nanobots, y la transformación somática y la inmortalidad. Como O'Mathuna da cuenta en cierto modo superficial, las dos categorías se borran mutuamente y los practicantes de cada una hacen uso extensivo de los practicantes y la retórica del otro. (Mody, 2011: 436)

El problema con el grueso de las perspectivas de la nanoética es que casi toda la atención, y ciertamente toda la pasión, está dirigida hacia el futurista "¿y si?" que hacia el normal “iqué es?” (Mody, 2011: 437).

Para ir incluso más a fondo, me permito agregar una tercera categoría a la nanoética normal y futurística: "la ética de la ficción”, corolario ético de la ciencia ficción.

Para regresar a la hipótesis de la plaga gris, Richard Smalley, galardonado en 1997 con el Premio Nobel de Química y aficionado temprano de las nanotecnologías, replicó a la pregunta sobre qué tan pronto serían realidad los robots a nanoescala señalando que "simplemente, nunca" (Smalley, 2001: 76). Obiamente, ésta es aún una pregunta controversial y muchos no concuerdan con Smalley, siendo el más famoso Erik Drexler (Drexler, 1986). El punto de este trabajo no es desde luego zanjar la disputa y decir quién está en lo correcto desde una perspectiva nanotecnológica molecular sino más bien enfatizar las profundas incertezas acerca de los avances de las nanotecnologías. Es entonces una pregunta relevante, en este contexto, el si los éticistas deberían o no invertir su tiempo considerando un caso que tal vez nunca ocurra - la distinción entre nanoética futurística siendo ésta la que refiere a desarrollos tecnológicos que podrían suceder o al menos considerados no como totalmente imposibles por una comunidad de expertos bien informados y que sí sería el caso de la ética ficción-.

\section{TIERRAS RARAS Y ÉTICA: ¿PRESENTE... E INEXISTENTE?}

El trazo de un paralelismo entre nanotecnologías y las tierras raras podría de algún modo parecer forzado. Los vínculos entre los dos casos existen, tal y como se desarrolla en la sección 3, pero eso no es esencial para nuestro propósito ahora. Las tierras raras usadas como ejemplo sintomático de un material que es esencia para nuestra modernidad $^{3}$ y que sin embargo ha generado hasta ahora poco interés entre los eticistas y los académicos del campo de los estudios Ciencia, Tecnología y Sociedad (STS, por sus siglas en inglés). Pese a ello, delinearé tres aspectos que son de interés para su análisis ético.

A.El daño y beneficio ambiental: ¿sacrificar el presente por un mejor futuro? La creciente parte de las aplicaciones de las tierras raras caen bajo la categoría de "tecnologías verdes": desde turbinas eólicas hasta focos ahorradores de energía, de autos híbridos a baterías recargables. Al mismo tiempo, los proce-

\footnotetext{
Las aplicaciones de las tierras raras son numerosas en múltiples campos que pueden ser vistos como el núcleo de nuestra modernidad: la producción de energía (por ejemplo, en la refinación de petróleo, motores eléctricos, vehículos híbridos, turbinas eólicas), productos de alta tecnología (componentes de hardware para computadoras portátiles y teléfonos celulares, pantallas de color para televisores de pantalla plana, etc.), la investigación médica (por mencionar algunos, catalizadores para la investigación química), entre muchos otros.
} 
sos de minado y procesamiento de los elementos de las tierras raras acarrean serios aspectos ambientales, posiblemente provocando problemas a la salud para la población concerniente. Este dilema lleva algunas veces a las siguientes retóricas: "soportemos las consecuencias negativas actuales para un mejor futuro" - no sobra decir que un futuro mejor es también visto en términos de crecimiento económico y oportunidades de trabajo-. Ésa fue, por caso, la retórica desarrollada por la Academia de Ciencias de Malasia en un informe en el que se comienza insistiendo sobre los riesgos globales de calentamiento global y la necesidad de desarrollar tecnologías verdes para así justificar una nueva orientación de la industria de las tierras raras.

"Malasia se coloca, por tanto, estratégicamente, en el desarrollo de sus industrias de tecnologías verdes, al contribuir así a las economías de bajo carbono alrededor del mundo y ayudando a asegurar un planeta sustentable para las generaciones futuras" (ASM, 2011: vii).

La pregunta, no obstante, se mantiene: ¿están sirviendo a las generaciones futuras los mineros de Baotou ${ }^{4}$ o los trabajadores de Malasia, o más bien a los inconformistas europeos y estadounidenses que podrían ser sus hijos ahora? La conciencia ética requiere ser cuidadoso en relación a que un discurso intergeneracional no sirve para esconder, de hecho, un asunto actual de injusticia intrageneracional.

B. El monopolio Chino y la dependencia occidental: ¿quién es dueño de qué?

Un aspecto planteado en relación con los elementos de tierras raras es la obligación legal - y moral- de China de exportar sus recursos. Es bien sabido el hecho de que China produce actualmente más del 95\% de las tierras raras a nivel mundial. Lo que es menos mencionado es que el país "sólo" tiene un tercio de las reservas conocidas - es decir, que la suerte geológica no lo explica todo-. Pese a ello, los países que son dependientes de las exportaciones de China han hecho quejas formales ante la OMC acerca de las cuotas de exportación chinas. ${ }^{5}$ Es un caso complejo, basado en muchas sutilezas - como la interpretación de los artículos del GATT referentes a la protección del ambiente lo que no es propósito ahora de revisión (mayores detalles en Verrax, 2013)-. Aun así, uno podría plantear la pregunta no en términos legales y de obligaciones contractuales sino más bien en términos de aventuras éticas: ¿hay algún tipo de obligación moral, un proceso de exploración para países con recursos pobres? Y para aquellos como Estados Unidos o Australia, con documentados depósitos de tierras raras, ¿hay algún tipo de obligación de "minar en casa" primero, ${ }^{6}$ es decir, de soportar también asumir el daño ambiental?

C. Usos competitivos

Finalmente, tal y como ya se mencionó, las tierras raras tienen muchas aplicaciones, incluyendo las de la industria militar tanto como las de la investigación médica — sólo por mencionar un par de casos de áreas opuestas en el imaginario popular-. En tiempo de reconocida escasez — si no física, al menos econó-

4 En Baotou (Mongolia, China) se localiza la mina más grande del mundo de tierras raras.

Me refiero al caso registrado conjuntamente en marzo de 2012 por EUA (DS431), la Unión Europea (DS432) y Japón (DS433).

6 En años recientes, muchos país se han involucrado en procesos de exploración —Japón, especialmente, cuya dependencia en exportaciones chinas es total-. 
mica- ¿quién se adjudica el derecho a decidir qué usos deberían priorizarse? ¿Debería haber algún tipo de regulación vertical (de arriba hacia abajo) planificada para distribuir los recursos por sector industrial? ¿Deberían los ciudadanos tener la palabra en términos de preferencias?

Como se puede observar, éstas son preguntas bien aterrizadas reflejadas en una situación presente y, sus respuestas podrían tener, de hecho, un impacto en el manejo de tales metales a muy corto plazo. Por tanto, básicamente esto sería lo opuesto a la nanoética que tiende a enfocarse más en escenarios futuros (si no es que en aquellos de ética ficción) y hace un llamado por una decisión no específica porque no está tratando con ningún asunto real actual.

Por tanto, cabe plantearse si están estos dos campos conectados a pesar de parecer estar tan alejados y en su caso cómo.

\section{NANOTECNOLOGÍAS Y LOS ELEMENTOS DE TIERRAS RARAS}

\section{Sobre cómo están conectados en el mundo material}

La misma idea de comparar las nanotecnologías con los elementos de tierras raras podría sorprender en una primera impresión: mientras las primeras pueden ser definidas por la escala en la que una tecnología dada opera que, además, podría involucrar prácticamente cualquier tipo de desarrollo tecnológico, los segundos representan una familia de elementos químicos con propiedades similares. ${ }^{7}$ Los vínculos entre las nanotecnologías y los elementos de tierras raras pueden observarse en dos sentidos: las tierras raras son empleadas en muchos desarrollos nanotecnológicos; al mismo tiempo, las nanotecnologías son vistas como una forma de mitigar la demanda de tierras raras.

Por un lado, el primer aspecto puede ser revisado exhaustivamente en un libro reciente (Tan, 2012) que ofrece una revisión integral de los diferentes usos de las tierras raras en las nanotecnologías, desde su uso para nanomateriales en LEDs, hasta su uso en la generación de imágenes fluorescentes y por resonancia magnética (MRI, por sus siglas en inglés).

Por el otro lado, puede sostenerse que las alternativas que permiten las nanotecnologías podrían mitigar la presión sobre el mercado de tierras raras. La nanotexturización de materiales electrodo podría, en efecto, ser usada para mejorar la tecnología actual de iones de litio. En relación con una de las principales aplicaciones de tierras raras, los magnetos, la mezcla de nanopartículas de tierras raras con nanopartículas de hierro, podría producir magnetos tan potentes con mucha menor cantidad de tierras raras incorporadas.

Como se ha visto, las nanotecnologías no remplazarán las tierras raras sino, más bien, permitirán disminuir la cantidad de su uso. Los artefactos creados con base en nanopartículas de tierras raras ¿deberían, entonces, seguir siendo analizados a través del prisma de la nanoética?

\footnotetext{
El término "tierras raras" se refiere a 17 elementos químicos: la familia de los lantánidos (con número ató-
} mico entre 57 y 71), el escandio y el itrio (dadas sus propiedades magnéticas similares a los lantánidos). 


\section{Similitudes éticas}

En ambos casos el enfoque sobre potenciales riesgos y beneficios tiende a dominar los discursos éticos tal y como ha sido advertido por varios autores (véase, por ejemplo, Strand y Nydal, 2008). Esto nos lleva a muchas formulaciones maniqueas tales como:

Claramente, la tecnología puede producir beneficios como daños. En particular, la nanotecnología ofrece mucho más esperanza para mejorar la condición humana. (Moor y Weckert, 2004: 304)

Ejemplos tomados por tales autores incluyen la limpieza del entorno natural a través del diseño de fotosíntesis artificial y, por tanto, de la manufactura a bajo costo de agua potable y alimentos seguros, la pelea del cáncer mediante el uso de nanobots, etcétera. Como los autores o exponen:

[...] una tecnología que ofrece la esperanza de un ambiente más limpio, mejores materiales, mejorar la salud, abundancia de alimentos, y computadoras más económicas, resulta muy atractiva. (Ibid.)

Tan entusiastas como son, los autores reconocen, asimismo, que "los potenciales beneficios son inmensos, pero los potenciales riesgos son inmensos también" (Ibid.: 305). Así, de acuerdo con los mencionados autores, dado que “...los resultados dañinos son inevitables, la nanoética será necesaria”. Es interesante observar, que en gran mediad basan la necesidad de la nanoética en la predicción de resultados negativos, posicionándose a sí mismos en el reino de lo que Johnson (2007) califica como "ética negativa".

El problema de tal perspectiva es dar la impresión de que los resultados positivos y negativos son en cierto sentido conmensurables y, por tanto, que un clásico análisis costo beneficio puede resolver todas las cuestiones. Aún más, los resultados negativos son usualmente fraseados en términos de "riesgo". Y como bien lo ha señalado Jacques Bordé, ${ }^{8}$ el asunto del riesgo es muy diferente del de la ética. La ética es cualitativa, el riesgo, por el contrario, es una noción cuantitativa. ${ }^{9}$

El riesgo de enfocarse mucho en el riesgo y la dicotomía maniquea entre resultados "buenos" y "malos" es que nos limita a una noción costo-beneficio consecuencialista al tiempo que se pierden de vista alternativas potenciales muy fructíferas.

\section{Contrastes éticos}

El principal contraste parece ser uno de temporalidad: mientras al tratar con tierras raras algunos aspectos pueden descomponerse en "ignorar el presente para un mejor futuro", en el caso de la nanoética, como se ha dicho, parece que los eticistas están casi siempre fantaseando sobre catástrofes futuras e ignorando los beneficios modestos actuales.

\footnotetext{
8 Bordé dio pie a la iniciativa para realizar una guía ética en el marco del comité de nanoética, parte del comité de ética del Centro Nacional de Investigaciones. Véase: <www.cnrs.fr/comets/IMG/pdf/10-ethique-nanos. pdf>.

9 Textual: “La question du risque est très différente de celle de l'éthique. L'éthique est qualitative, le risque, au contraire, est une notion quantitative".
} 
Hasta ahora, ninguna vida humana ha sido amenazada con el uso de las nanotecnologías. Contrariamente, el minado y procesamiento de tierras raras suponen haber causado ${ }^{10}$ y siguen causando muchos problemas de salud entre los mineros y la población local, todo a la par que se han contaminado los suelos y aguas alrededor de Baotou.

Enfocarse en un futuro incierto en lugar de hacerlo en el presente es problemático por varias razones. Puede inducir, por ejemplo, el riesgo, o puede ser el síntoma de lo que se ha calificado como esquizofrenia moral.

No conmoverse por lo que uno valora - lo que uno considera bueno, agradable, correcto, hermoso, y así sucesivamente- nos habla de una enfermedad del espíritu. No valorar lo que nos conmueve también nos habla de una enfermedad del espíritu. Tal enfermedad, o enfermedades, puede ser adecuadamente calificada como "esquizofrenia moral" —porque son una división entre los motivos de uno y las razones de uno. (Stocker, 1976: 453-454)

Ignorar lo que uno conoce mejor, el presente, y enfocarse en incertezas futuras, puede ser visto como una ilustración de tal esquizofrenia moral.

A continuación delinearé una alternativa ética que se espera pueda ser útil para hacer frente a la esquizofrenia moral que surge de la dicotomía temporal. Pero, en principio, es necesario comprender aquello que interesa a la imperante clase de ética del " ¿y si?”.

\section{HACIA UN GIRO ÉTICO SITUADO, INCORPORADO Y ORIENTADO EN EL PRESENTE}

Sugiero dos razones que explican la tendencia de la ética para enfocarse en aspectos aún no ocurridos y que tal vez nunca lo hagan. La primera surge del impulso humano por imaginar y prever un futuro con tendencias a verse mucho más interesante de lo que de hecho sabemos y experimentamos en nuestras vidas actuales. El segundo proviene del miedo, del miedo a ser normativo y responsable. De hecho, si los eticistas dejaran de escribir en un estilo propio de la esfera protectora del “¿y si?”, se les solicitaría que dieran una opinión real sobre un aspecto actual y que consideren ser responsables de sus elecciones.

De hecho, la perspectiva temporal es sintomática de otra falacia que podemos llamar "ética abstracta". ${ }^{11} \mathrm{Si}$ aceptamos el punto de vista contextualizado —en oposición al punto de vista moral- los juicios éticos casi siempre ocurren cuando se incorporan en situaciones de la vida real:

[...] las situaciones en que ejercitamos nuestra experiencia ética sobrepasa por mucho aquellas en las que debemos ejercitar la deliberación ética explícita. (Varela, 1999: 23)

Por tanto, localizar el debate acerca del desarrollo de una tecnología específica en el futuro acarrea dos inconvenientes. Primero y de modo paradójico, hace la discusión más moralística y normativa para las peculiaridades contextuales no consideradas. Segundo, permite a los eticistas rechazar la rendición de cuentas.

\footnotetext{
${ }^{10}$ Ha sido muy delicado y difícil el acceso a información confiable de parte de la República Popular China.

${ }^{11}$ Varela (1999) se pregunta "por qué uno podría confundir el comportamiento ético con el juicio", distinguiendo así entre lo que él llama la tradición abstracta cartesiana occidental y un nuevo paradigma basado en la idea de que las unidades de conocimiento son concretas. Aboga, por tanto, por un modelo nuevo que denomina "cognición como enacción" (cognition as enaction).
} 
La tabla 1 resume las dos visiones discutidas aquí.

TABLA 1. Visión moralizada versus visión contextualizada

\begin{tabular}{|l|l|l|}
\hline & \multicolumn{1}{|c|}{ Moralidad } & \multicolumn{1}{c|}{ Contextualización } \\
\hline ¿Cómo? (proceso) & $\begin{array}{l}\text { Análisis } \\
\text { Deliberación }\end{array}$ & $\begin{array}{l}\text { Promulgación } \\
\text { Encarnación / Personificación }\end{array}$ \\
\hline ¿Qué? (herramientas éticas) & $\begin{array}{l}\text { Escenarios } \\
\text { Experimentos de pensamiento } \\
\text { "ética de ficción" }\end{array}$ & $\begin{array}{l}\text { Descripción de la realidad } \\
\text { Fenomenología }\end{array}$ \\
\hline $\begin{array}{l}\text { ¿Quién? (alcance de la } \\
\text { expertise) }\end{array}$ & $\begin{array}{l}\text { Expertos académicos y } \\
\text { técnicos }\end{array}$ & $\begin{array}{l}\text { Cualquiera es un experto en } \\
\text { ética }\end{array}$ \\
\hline $\begin{array}{l}\text { Tipo de acción } \\
\text { Consciente e intencional }\end{array}$ & $\begin{array}{l}\text { Desinteresado y no } \\
\text { intencional }\end{array}$ \\
\hline Principales autores & $\begin{array}{l}\text { Kant, Habermas, Rawls, } \\
\text { Maclntyre }\end{array}$ & \begin{tabular}{l} 
Hegel, Taylor, Varela, Puech \\
\hline
\end{tabular} \\
\hline
\end{tabular}

Fuente: Elaboración propia.

Cabe aclarar que en la visión contextualizada cualquiera es un experto en tanto pertenezca a la comunidad humana:

[...] todos somos expertos porque pertenecemos a una tradición textualizada en la que nos movemos fácilmente. (Varela, 1999: 24).

En contraste con la creciente ética de ficción, a continuación se expone una agenda posible para la investigación desde una perspectiva de ética situada en ocuparse de los desarrollos tecnológicos más novedosos como las tierras raras, las nanotecnologías y otras.

En primer lugar, esta agenda implica asumir nuestro poder tecnológico y extraer las consecuencias de ello. Esto no quiere decir confiar en abstracto, descontextualizados - léase "directrices éticas" o "códigos de conducta" que algunos especialistas están tan interesados en producir- $-{ }^{12}$ Ello significa ser consciente de la textura de este nuevo contexto tecnológico para que nosotros - los ciudadanos, los científicos, los consumidores que tienen que lidiar con los nuevos productos y tecnologías- podamos identificar situaciones éticas potenciales.

En segundo lugar, asumir la responsabilidad de las compensaciones que son y serán cada vez más necesarias en tal novedoso contexto — que no quiere decir que las tecnologías sólo pueden ser definidas por sus lados "buenos" y "malos". En este punto vale recordar lo que se conoce como "la ley de Kranzberg": "la tecnología no es bue-

\footnotetext{
${ }^{12}$ A modo de ejemplo, citemos un extracto del código europeo de conducta para las nanociencias y nanotecnologías responsables: "las actividades de investigación en nanociencias y nanotecnologías deben ser comprensibles para el público. Deben respetar los derechos fundamentales y llevarse a cabo tanto bajo en su diseño como en su ejecución, difusión y uso, en el interés del bienestar de los individuos y la sociedad" (Comisión Europea, 2009: 14). Estos principios generales, en mi opinión, son inútiles para las acciones éticas, debido en parte a que no están en lo absoluto integrados en un contexto peculiar.
} 
na ni mala, sino neutral" (Kranzberg, 1986). Obviamente es el carácter neutral lo que es importante aquí porque más allá de la evaluación moral, los avances tecnológicos sí cambian nuestro mundo, el contexto de nuestras acciones, y por esa razón, nunca son éticamente neutrales.

"Esto está muy bien" podría estar pensando incluso el lector más benévolo, pero ¿cómo sería concretamente una nanoética situada y actualmente orientada? Estamos tan acostumbrados a pensar en la ética en términos de futuro, en términos de potenciales problemas, que podríamos tener problemas para identificar aquellos que están justo frente a nosotros. Hace un par de años, León Olivé identificaba, en esta misma revista, un problema ético fundamental de la tecnociencia:

Un problema ético fundamental planteado en relación con la tecnociencia es el de si es posible lograr normas de convivencia armoniosa y de resolución pacífica de conflictos, dado que tales sistemas afectan a muy diversos grupos con intereses y valores diferentes. (Olivé, 2009: 56)

El tema de la "coexistencia pacífica" entre los grupos y las comunidades cuyos valores e intereses son divergentes, si no es que incompatibles, ya es algo muy relevante en el mundo en que actualmente vivimos. No es el propósito de este artículo trazar una agenda de investigación exhaustiva para el nuevo enfoque ético que propongo, pero voy a adentrarme con un poco más de detalle con una ilustración. Los aspectos éticos que son usualmente mencionados en torno a la discusión de la nanoética tienen qué ver con la posibilidad de ampliar enormemente la esperanza de vida - de acuerdo con el nanoentusiasta y futurista Ray Kurzweil-, en realidad, casi acercándose a la inmortalidad.

Es la extensión de vida radical. La plena realización de nanobots, básicamente, permitirá eliminar la enfermedad y el envejecimiento biológico. Creo que en 20 años vamos a ver el uso generalizado de nanodispositivos que realizan ciertas funciones para nosotros. En 30 o 40 años vamos a superar la enfermedad y el envejecimiento. Los nanobots podrán explorar órganos y células que necesitan reparaciones y simplemente los arreglarán. Esto dará lugar a profundas extensiones a nuestra salud y longevidad. (Gaudin, sin fecha)

Mi punto aquí no es decir que los avances nanotecnológicos no harán este asunto aún más preciso en el futuro, sino más bien que ya los estamos enfrentando en nuestro mundo actual. Las discrepancias con la esperanza de vida entre los países y dentro de un mismo país, entre las comunidades de bajos ingresos y altos ingresos, o entre diferentes grupos éticos, que ya es hoy por hoy terrible (menos de 40 años en el África subsahariana de cara a más de 80 años en la mayoría de países europeos, Australia, Japón y Canadá). Sí, el uso de las nanotecnologías aumentaría la brecha aún más, pero y ¿qué estamos haciendo para atender el problema de la discrepancia de vida hoy día? ¿No es acaso ésa una cuestión ética?

\section{Conclusión}

En este artículo he argumentado que otra forma de abordar los problemas éticos derivados del desarrollo de las nanotecnologías es posible y deseable. Esto es por supuesto aplicable al campo de la "tecnociencia" en general, dígase las nanotecnologías, 
la geoingeniería o las biotecnologías en tanto que todas comparten la particularidad de ser tratadas por parte de los especialistas en ética, esencialmente desde una perspectiva futurista.

Las principales consecuencias prácticas de este cambio de paradigma serían, tanto la cesantía de directrices éticas generales o códigos de conducta ya que no están integrados en un contexto particular, como la apuesta por la participación generalizada de los ciudadanos y los consumidores en las deliberaciones públicas — no como personas comunes, sino como "expertos éticos".

\section{REFERENCIAS BIBLIOGRÁFICAS}

ASM (2011) Rare earth industries: moving malaysia's green economy forward. <http://dspace.uniten.edu.my/jspui/bitstream/123456789/4002/1/RARE\%20EARTH\%20_ Report_English.pdf>.

Comisión Europea (2009) Commision recommendation on a code of conduct for responsible nanosciences and nanotechnologies research \& Council conclusions on responsible nanosciences and nanotechnologies research. <http://ec.europa.eu/research/sciencesociety/document_library/pdf_06/nanocode-apr09_en.pdf>.

Drexler, K. E. (1986) Engines of creation. The coming era of nanotechnology. Doubleday.

Gaudin, S. (sin fecha) "Nanotech could make humans inmortal by 2040, futurist says". <www.computerworld.com/s/article/9138726/Nanotech_could_make_humans_ immortal_by_2040_futurist_says $>$.

Godman, M. (2008) "But is it unique to nanotechnologies? Reframing Nanoethics". Science and Engineering Ethics, vol. 14, núm. 3: 391-403.

Grunwald, A. (2005) “Nanotechnology - A new field of ethical inquiry?”. Science and Engineering Ethics, vol. 11: 187-201.

Jasanoff, S. (2004) States of knowledge: The co-production of science and the social order. Londres, Reino Unido: Routledge.

Johnson, D.G. (2007) “Ethics and technology 'in the making': An essay on the challenge of nanoethics". Nanoethics, vol. 1: 21-30.

Kranzberg, M. (1986). “Technology and History: "Kranzberg's Laws"”. Technology and Culture, vol. 27, núm. 3: 544-560.

Mody, Cyrus C.M. (2011) "Nanoethics: Big ethical issues with small technology (review)", Technology and Culture, vol. 52, núm. 2: 436-437.

Moor, J., Weckert, J. (2004) "Nanoethics: Assessing the nanoscale from an ethical point of view”: En i Baird, D., Nordmann, A., Schummer, J. (eds.). Discovering the nanoscale. Ámsterdam: IOS Press.

Nurock, V. (2010) “Nanoethics: Ethics for, from, or with nanotechnologies?”. HYLE - International Journal for Philosophy of Chemistry, vol. 16: 31-42.

Olivé, L. (2009) "Problemas axiológicos y éticos de la tecnociencia". MundoNano, vol. 1, núm. 2: 48-60.

O'Mathuna, D. P. (2009) Nanoethics: Big ethical issues with small technology. Continuum.

Puech, M. (2013) "Ordinary technoethics”, International Journal of Technoethics, vol. 4, núm. 2: 36-45. 
Ruivenkamp, M. y Rip, A. (2012) "Entanglement of imaging and imagining of nanotechnology”, Nano Ethics, vol. 5, núm. 2: 185-193.

Smalley, R. E. (2001) “Of chemistry, love, and nanobots”. Scientific American, 285: 76-77.

Stocker, M. (1976) "The schizophrenia of modern ethical theories". The Journal of Philosophy, vol. 73, núm. 14. On motives and morals: 453-466.

Strand, R., Nydal R. (2008) “Nanoética buena - Nanotecnología buena”, MundoNano, vol. 1, núm.1: 61-79.

Tan, T. T.Y. (2012) Rare earth nanotechnology. Pan Stanford Publishing.

Varela, F. J. (1999) Ethical know-how: Action, wisdom, and cognition. Writing Science, Stanford University Press.

Verrax, F. (2013) Making sense of rare earth elements: An interdisciplinary approach to mineral ressources' ethics and governance, tesis doctoral. Noruega: Universidad de Bergen. 